\title{
Effect of salbutamol on innervated and denervated rat soleus muscle
}

T. Šoić-Vranić, D. Bobinac, S. Bajek, R. Jerković,

D. Malnar-Dragojević and M. Nikolić
Department of Anatomy, Medical Faculty, University of Rijeka, Rijeka, Croatia
Correspondence

T. Šoić-Vranić

Department of Anatomy

Medical Faculty

University of Rijeka

Braće Branchetta 20,

51000 Rijeka

Croatia

E-mail: tamaras@medri.hr ......................

Received September 29, 2004

Accepted June 1, 2005

\begin{abstract}
The objective of the present investigation was to perform a 14-day time-course study of treatment with salbutamol, a $\beta_{2}$ adrenoceptor agonist, on rat soleus muscle in order to assess fiber type selectivity in the hypertrophic response and fiber type composition. Male Wistar rats were divided into four groups: control $(\mathrm{N}=10)$, treated with salbutamol $(\mathrm{N}=30)$, denervated $(\mathrm{N}=30)$, and treated with salbutamol after denervation $(\mathrm{N}=30)$. Salbutamol was injected intraperitoneally in the rats of the $2 \mathrm{nd}$ and 4 th groups at a concentration of $0.3 \mathrm{mg} / \mathrm{kg}$ twice a day for 2 weeks. The muscles were denervated using the crush method with pean. The animals were sacrificed 3, 6, 9, 12, and 14 days after treatment. Frozen cross-sections of soleus muscle were stained for myosin ATPase, $\mathrm{pH}$ 9.4. Cross-sectional area and percent of muscle fibers were analyzed morphometrically by computerized image analysis. Treatment with salbutamol induced hypertrophy of all fiber types and a higher percentage of type II fibers (21\%) in the healthy rat soleus muscle. Denervation caused marked atrophy of all fibers and conversion from type I to type II muscle fibers. Denervated muscles treated with salbutamol showed a significantly larger crosssectional area of type I muscle fibers, $28.2 \%$ compared to the denervated untreated muscle. Moreover, the number of type I fibers was increased. These results indicate that administration of salbutamol is able to induce changes in cross-sectional area and fiber type distribution in the early phase of treatment. Since denervation-induced atrophy and conversion from type I to type II fibers were improved by salbutamol treatment we propose that salbutamol, like other $\beta_{2}$ adrenoceptor agonists, may have a therapeutic potential in improving the condition of skeletal muscle after denervation.
\end{abstract}

\section{Introduction}

Salbutamol is a $\beta_{2}$ adrenoceptor agonist (BAA) known to induce bronchiolar relaxation, higher vascularization of skeletal muscle, as well as muscle hypertrophy (1). Martineau et al. (2) demonstrated that shortterm administration of salbutamol increases
Key words

- Rat skeletal muscle

- Soleus muscle

- Denervation

- Histochemistry

- Muscle fibers
- Salbutamol 
also demonstrated in skeletal muscles with altered physiological conditions or functional demands. Zeman et al. (5) showed that clenbuterol, another BAA, is able to retard muscle atrophy in denervated muscles. Clenbuterol treatment reduced the loss of wet weight and of protein content and also increased the cross-sectional area of denervated solei. Data reported by Maltin et al. (6) indicate that clenbuterol not only inhibits but also partially reverses denervation-induced atrophy of rat soleus muscle. This was evident from measurements of both muscle protein content and fiber cross-sectional areas. Furthermore, the hypertrophic effect of BAA seems to be selective for certain fiber types. Most studies have demonstrated that BAA increase the cross-sectional area of fast muscle fibers (7-9) while the results of studies of the effects of BAA on slow muscle fibers have not been consistent. Some studies have demonstrated that the cross-sectional area of slow twitch oxidative fibers is not changed by chronic administration of $\operatorname{BAA}(8,10,11)$ while others have shown that all fiber types are affected equally $(12,13)$. Recent reports have established that clenbuterol is able to induce changes in fiber type distribution. After 2 weeks of clenbuterol treatment there was a pronounced change in fiber type distribution, i.e., slow-to-fast transitions (7). Lynch et al. (14) have demonstrated slow-to-fast transformation in rat soleus muscle after 15 weeks of clenbuterol administration. Zeman et al. (8) have shown that a treatment period of more than 2 weeks was required to increase fast-twitch fiber composition. On the other hand, after four weeks of clenbuterol administration there were no significant fiber type alterations in tibialis anterior and intercostal muscles which are fast-twitch contracting muscles (15).

The aim of the present investigation was to perform a 14-day time-course study of salbutamol administration with two main objectives: 1) to elucidate the fiber type selectivity in the hypertrophic response and
2) to explore the changes in fiber type distribution in slow rat skeletal muscle, both in the presence and in the absence of the nerve.

\section{Material and Methods}

\section{Animals}

We used 3-month-old male Wistar rats weighing approximately 200-250 g ( $\mathrm{N}=$ 100 ), with free access to standard laboratory food and water. The animals were divided into four groups as follows: 1$)$ control $(\mathrm{N}=$ $10), 2)$ treated with salbutamol $(\mathrm{N}=30), 3)$ denervated $(\mathrm{N}=30)$, and 4) treated with salbutamol after denervation $(\mathrm{N}=30)$.

\section{Surgical procedure and treatment with salbutamol}

The animals from the 3rd and 4th groups were anesthetized with an intraperitoneal injection of ketamine hydroxychloride (25 $\mathrm{mg} / \mathrm{kg}, 1 \mathrm{mg} / \mathrm{ml}$ ketamine; Parke-Davis, Milan, Italy). The right sciatic nerve of the thigh was exposed and crushed proximal to its branching for denervation, with pean lasting $2 \mathrm{~min}$. The overlying muscles and skin were then sutured. Salbutamol (Pliva, Zagreb, Croatia), diluted in $0.3 \mathrm{mg} / \mathrm{kg} 0.9 \% \mathrm{NaCl}$, was injected intraperitoneally in the rats of the 2 nd and 4th groups twice a day for 2 weeks. The first salbutamol dose was injected $1 \mathrm{~h}$ after the surgical procedure.

Animals from the control group were analyzed as a zero time control. The animals from the other groups (salbutamol, denervated and denervated plus salbutamol) were then sacrificed by cervical hyperextension $3,6,9,12$, and 14 days after denervation. The soleus muscles were dissected from tendon to tendon from the right hindlimb.

\section{Muscle analysis}

Muscle specimens were frozen by immersion in liquid nitrogen and oriented as an 
upright cylinder in embedding medium (Tissue-Tek, Sakura, Netherlands) while still frozen. Serial $8-\mu \mathrm{m}$ thick transverse sections were cut with a cryostat at $-20^{\circ} \mathrm{C}$, attached to glass slides and stained for myosin ATPase, pH $9.4(16,17)$. Sections were then fixed at $40^{\circ} \mathrm{C}$ for $5 \mathrm{~min}$ in $20 \%(\mathrm{v} / \mathrm{v})$ paraformaldehyde, $0.1 \mathrm{M}$ sodium cacodylic acid containing $0.4 \mathrm{M}$ saccharose and rinsed well with running tap water. Rinsed sections were incubated at $37^{\circ} \mathrm{C}$ for $30 \mathrm{~min}$ in the following solution: $5 \mathrm{mg}$ ATP was dissolved in a few drops of distilled water, $10 \mathrm{ml} 0.2 \mathrm{M}$ sodium barbiturate buffer containing $0.1 \mathrm{M} \mathrm{CaCl}_{2}$ in distilled water was added and the $\mathrm{pH}$ was adjusted to 9.4. After washing with distilled water, sections were immersed in $2 \% \mathrm{CaCl}_{2}$ for three rinses of $1 \mathrm{~min}$ each. Specimens were rinsed again in distilled water and immersed in diluted (1:10) ammonium sulfide solution for $30 \mathrm{~s}$. After a final rinsing in running tap water the sections were dehydrated, cleared and mounted in glycerine.

In treated and untreated soleus muscles, fibers showing the densest reaction product were identified as fast-twitch oxidative glycolytic fibers, fibers showing no reaction product were identified as slow-twitch fibers, and fibers showing an intermediate reaction product were identified as fast-twitch glycolytic fibers. However, since fast-twitch glycolytic fibers represented less than $1.5 \%$ of all the fibers detected in the three experimental groups, i.e., treatment with salbutamol, denervation and denervation plus treatment with salbutamol, fast-twitch glycolytic fibers were excluded from the measurements and calculations as a separate group and were included in population of type II fibers. The cross-sectional areas of 200-300 fibers were measured with the SFORM image analyzer (VAMS, Zagreb, Croatia) in random fields of muscle sections obtained from each group. Counts of type I or type II fibers were used to calculate fiber type percentages. Data are reported as means $\pm \mathrm{SD}$. The control group was used for comparison with the healthy group treated with salbutamol and the denervated group was used for comparison with the denervated plus salbutamol group. Statistical analyses were performed by the Student $t$-test, with the level of significance set at $\mathrm{P}<0.05$.

\section{Results}

Changes in the cross-sectional area of muscle fibers

After 14 days of salbutamol administration, the cross-sectional areas of type I and type II muscle fibers were larger in healthy soleus muscle than in muscles of the untreated control group (Figures 1A,B and 2A,B). A statistically significant effect of salbutamol on fiber size was observed as

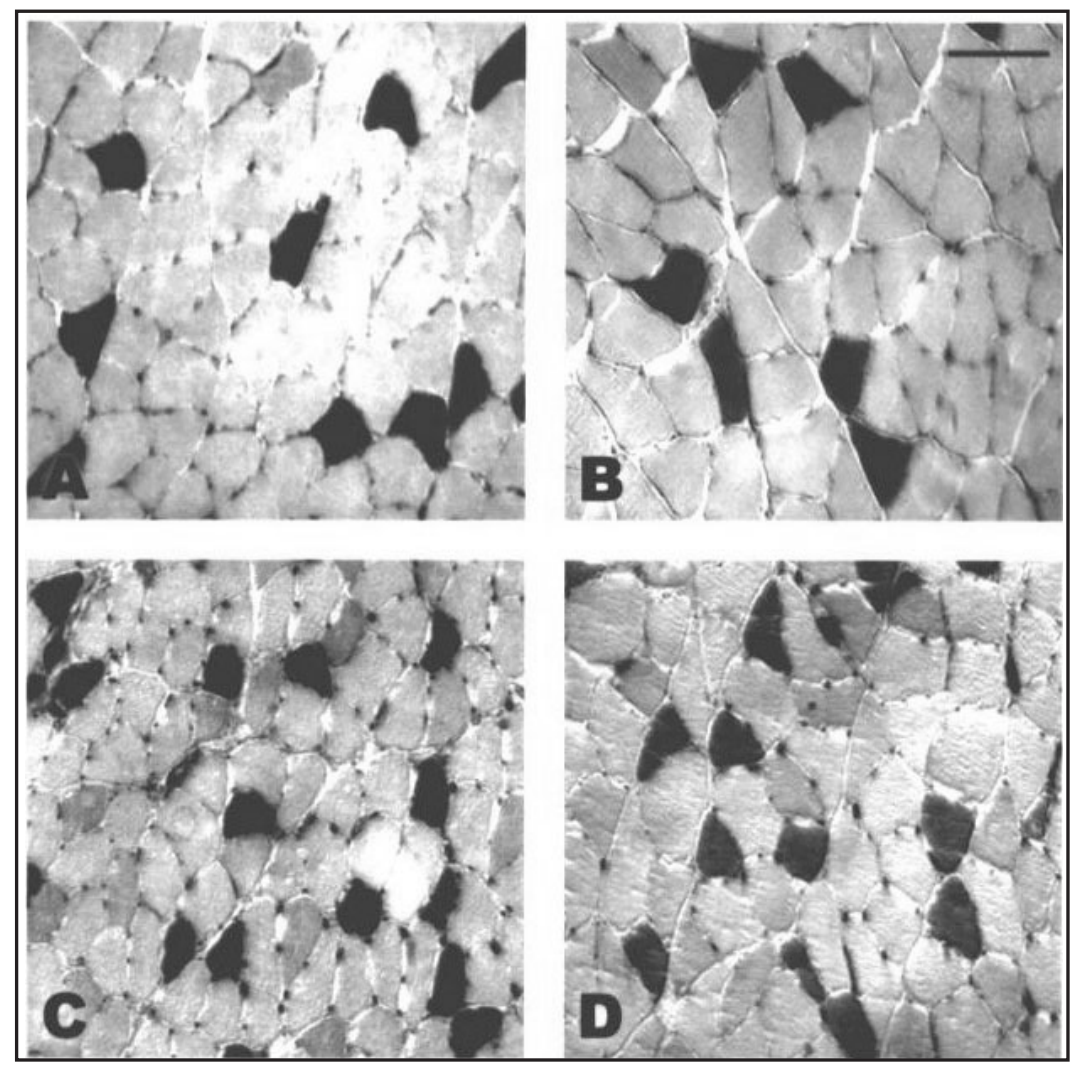

Figure 1. Rat soleus muscles stained for myosin ATPase at $\mathrm{pH} 9.4,14$ days after treatment with salbutamol. The dark color indicates type II fibers and the light color type I fibers. Panel $A$, Muscle fibers from the control group. Panel $B$, Muscle fibers after salbutamol treatment. Panel $C$, After denervation, muscle fibers had a smaller cross-sectional area. Panel $D$, Denervated muscle after treatment with salbutamol had an increased cross-sectional area. Bar $=100 \mu \mathrm{m}$ for all panels. 

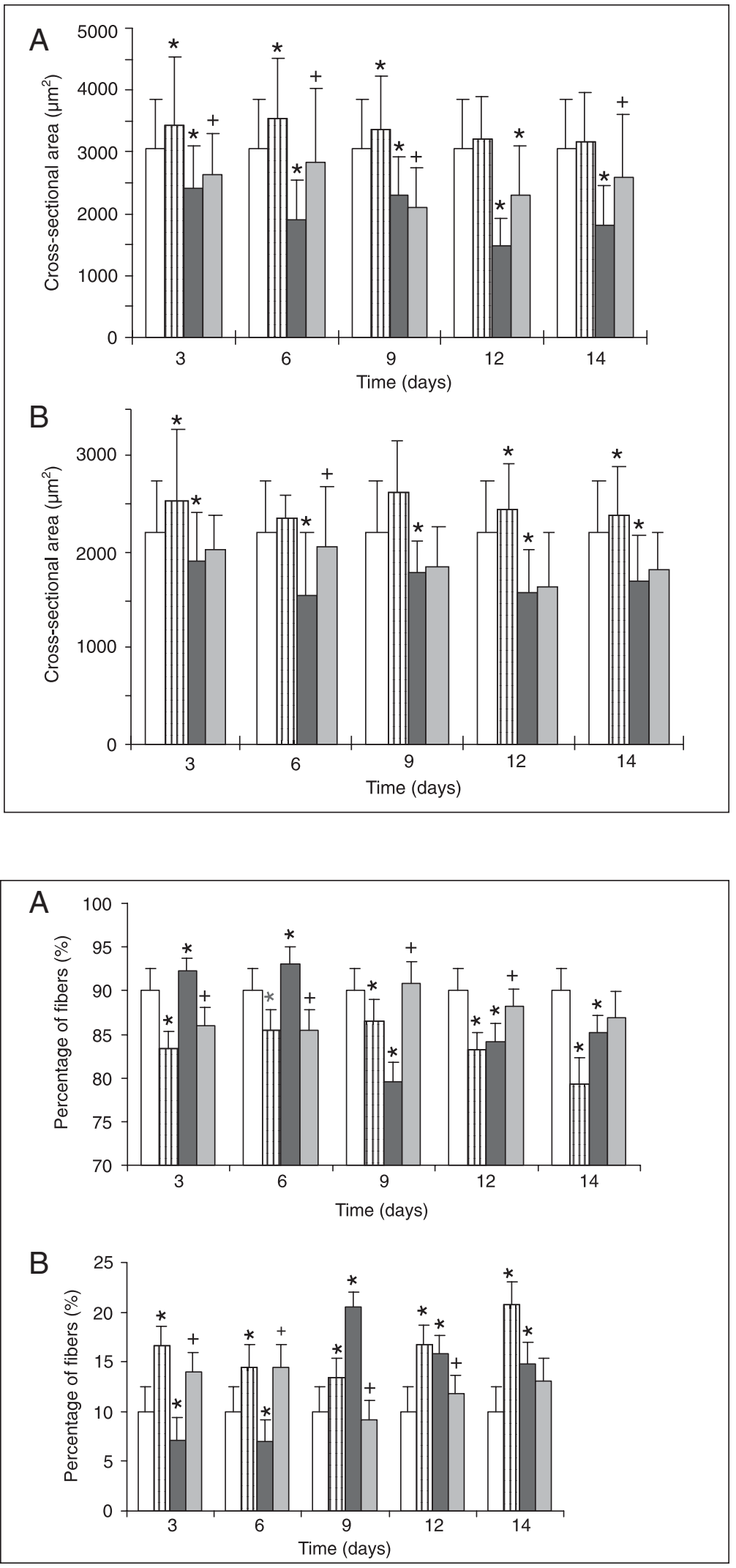

Figure 2. Changes in cross-sectional area of soleus muscle type I (A) and type II (B) fibers from the four treated groups. Open columns = control group; vertical lined columns = group treated with salbutamol; dark gray columns = denervated group; light gray columns = group treated with salbutamol following denervation. Data are reported as means \pm SD. ${ }^{*} P<0.05$ compared to control; ${ }^{+} \mathrm{P}<0.05$ compared to the denervated group (Student $t$-test).

Figure 3. Distribution of type I and II fibers in soleus muscle. $A$, Type I fibers from the four treated groups; $B$, type II fibers from the four treated groups. Open columns = control group; vertical lined columns = group treated with salbutamol; dark gray columns $=$ denervated group; light gray columns = group treated with salbutamol following denervation. ${ }^{*} \mathrm{P}<0.05$ compared to control; ${ }^{+} \mathrm{P}<0.05$ compared to the denervated group (Student $t$-test). 
early as 3 days after treatment $(\mathrm{P}<0.05)$. After denervation, the cross-sectional areas of both types of muscle fibers decreased significantly ( $\mathrm{P}<0.05$; Figures $1 \mathrm{C}$ and $2 \mathrm{~A}, \mathrm{~B})$. By the 14th day of denervation the crosssectional area of type I fibers was reduced to $59.4 \%$ and that of type II fibers was reduced to $6.7 \%$ compared to healthy muscle (Figure 2A,B). The cross-sectional area of type I fibers of denervated muscles treated with salbutamol increased from $1815.3 \pm 650.6$ to $2529.7 \pm 1018.1 \mu \mathrm{m}^{2}(\mathrm{P}<0.05)$, corresponding to a $28.24 \%$ increase compared to denervated untreated muscle $\left(1815.3 \pm 650.6 \mu \mathrm{m}^{2}\right)$ at the end of experiment (Figures 1C,D and 2A). Type II fibers of denervated muscles treated with salbutamol were not significantly affected although a transient increase of $24.03 \%$ in fiber size was observed on the 6th day $(\mathrm{P}<0.05)$.

\section{Changes in the proportions of muscle fiber types I and II}

Soleus, as a slow-twitch muscle, is composed of $90 \%$ type I fibers and 10\% type II fibers. The influence of salbutamol administration was observed in the early phase of the experiment starting on the 3rd day. In control muscle, salbutamol increased the number of type II fibers, from 10 to $21 \%$, a fact that was most evident by the 14th day of treatment $(21 \%$; $\mathrm{P}<0.05$; Figure $3 \mathrm{~B})$. The number of type II fibers increased from the 9th day of denervation $(\mathrm{P}<0.01$; Figure $3 \mathrm{~B})$ and was clearly demonstrable until the end of the experiment. Furthermore, a higher percentage of type II fibers was observed in denervated soleus during the first 6 days of salbutamol administration (Figure 3B). Salbutamol increased the percentage of type I fibers until the end of the experiment (Figure $3 \mathrm{~A})$.

\section{Discussion}

The aim of this investigation was to ex- plore the early effects of treatment with salbutamol, a BAA, on fiber size and fiber distribution in denervated and control rat soleus muscle. It is known that the soleus is made up of $90 \%$ type I fibers, which are slow-twitch oxidative, and $10 \%$ of type II fibers which are fast-twitch oxidative-glycolytic. In the present experiment, administration of salbutamol for 14 days resulted in an increase in cross-section of both type I and type II fibers. These changes were observed as early as 3 days after salbutamol administration. However, on day 14 the increase in cross-sectional area of type I fibers was only $3 \%$ compared to that of type II fibers, which was $8 \%$ in relation to untreated muscle. These results agree with those reported by others. Maltin et al. (13) and Zeman et al. (8) obtained the same result in soleus and extensor digitorum longus muscles after the administration of clenbuterol $(2 \mathrm{mg} / \mathrm{kg}$ in food and $1.3 \mathrm{mg} / \mathrm{kg}$ in drinking water, respectively) for 7 days. However, after the 3rd and the 12th weeks of their experiments, respectively, a significant increase in crosssectional area was observed only in type II but not in type I fibers. This fact is in accordance with the idea that fast-twitch fibers show greater plasticity in response to external factors that regulate muscle growth (13).

In the present experiment, during the denervation period the activity of salbutamol had a stronger influence on slow-twitch oxidative fibers. Maltin et al. (12) showed that the number of BAA receptors on oxidative fibers increases, triggering the process of increased protein synthesis. As soleus muscle is an oxidative muscle, it could be postulated that salbutamol has a specific effect in terms of hypertrophy, namely, it stops the process of muscle fiber atrophy during the denervation period. These changes occurring in denervated muscle fibers under the influence of salbutamol led Maltin et al. (18) to think of a classic response of cells to growth factors, with salbutamol acting as an anabolic drug as well as a $\beta$-agonist agent. 
After 2 weeks of salbutamol administration, we observed a more pronounced effect on fiber composition than on the anabolic response, in agreement with data reported by Ricart-Firinga et al. (7). Over 14 days the number of type II fibers increased significantly from the initial 10\% observed in healthy soleus up to $21 \%$, in agreement with data reported by Zeman et al. (8). This occurs also during mechanical unweighting $(10,19)$ and during the period of denervation. Actually, during muscle maturation the number of type I fibers in soleus increases at the expense of the number of type II fibers. Administration of clenbuterol prevents the conversion of type II fibers to type I, normally occurring in the process of maturation, due to the effect of the drug on increased synthesis and accumulation of fast myosin light chains in soleus (8). Stevens et al. (20) demonstrated that, during 2 weeks of administration, clenbuterol produced changes in the group of type II fibers. Treatment with clenbuterol causes the upregulation of IIA fibers (23\%) and, in addition, the induction, of considerable amounts of the faster IIX (3\%) and fastest IIB (2\%) fiber types. These data demonstrated the high plasticity of muscle spanning the spectrum of fiber types.

The number of type I fibers decreased during the first 6 days in denervated soleus treated with salbutamol compared to denervated untreated muscle. However, from the 6th day to the end of the experiment the number of type I fibers was higher in the former than in the latter. On day 9 the activity of salbutamol resulted in such a significant increase in the number of these fibers that it almost reached the corresponding value in control muscle. Incidentally, the same day marked the lowest value for type I fibers. This leads us to conclude that the most significant changes in fiber distribution occurred on day 9. Indeed, on day 9 there was the most profound conversion of type I to type II fibers in denervated muscles and of type II to type I in denervated muscles under the influence of salbutamol. Thus, we may postulate that salbutamol reestablishes the process of growth and development in denervated muscles with the occurrence of the process of conversion of type II to type I fibers (21).

\section{References}

1. Apperley GH, Daly MJ \& Levy GP (1976). Selectivity of B-adrenoceptor agonists and antagonists on bronchial, skeletal, vascular and cardiac muscle in the anesthetized cat. British Journal of Pharmacology, 57: 235-246.

2. Martineau L, Horan MA, Rothwell NJ et al. (1992). Salbutamol, a B $\mathbf{2}^{-}$ adrenoceptor agonist, increases skeletal muscle strength in young men. Clinical Science, 83: 615-621.

3. Emery PV, Rothwell NJ, Stock MJ et al. (1984). Chronic effects of Badrenergic agonists on body composition and protein synthesis in the rat. Bioscience Reports, 4: 83-91.

4. Li JB \& Jefferson LS (1977). Effect of isoproterenol on amino acid levels and protein turnover in skeletal muscle. American Journal of Physiology, 232: E243-E249.

5. Zeman RJ, Ludemann R \& Etlinger JD (1987). Clenbuterol, a beta2-agonist, retards atrophy in denervated muscles. American Journal of Physiology, 252: E152-E155.

6. Maltin CA, Reeds PJ, Delday MI et al. (1986). Inhibition and reversal of denervation induced atrophy by the beta-agonist growth promoter, clenbuterol. Bioscience Reports, 6: 811-818.
7. Ricart-Firinga C, Stevens L, Canu MH et al. (2000). Effects of $B_{2^{-}}$ agonist clenbuterol on biochemical and contractile properties of unloaded soleus fibers of rat. American Journal of Physiology, 278: C582-C588.

8. Zeman RJ, Ludemann R, Easton TG et al. (1988). Slow to fast alterations in skeletal muscle fibers caused by clenbuterol, a beta $2^{-}$ receptor agonist. American Journal of Physiology, 254: E726-E732.

9. Criswell DS, Powers SK \& Herb RA (1996). Clenbuterol-induced fiber type transition in the soleus of adult rats. European Journal of Applied Physiology, 74: 391-396.

10. Maltin CA, Delday MI, Hay SM et al. (1990). Effects of bovine pituitary growth hormone alone or in combination with the betaagonist clenbuterol on muscle growth and composition in veal calves. British Journal of Nutrition, 63: 535-545.

11. Miller MF, Garcia DK, Coleman ME et al. (1988). Adipose tissue, longissimus muscle and anterior pituitary growth and function in clenbuterol-fed heifers. Journal of Applied Physiology, 66: 12-20.

12. Maltin CA, Delday MI, Hay SM et al. (1992). Denervation increases clenbuterol sensitivity in muscle from young rats. Muscle and Nerve, 
14: 188-192

13. Maltin CA, Delday MI \& Reeds PJ (1986). The effect of a growth promoting drug, clenbuterol, on fiber frequency and area in hind limb muscles from young male rats. Bioscience Reports, 6: 293-299.

14. Lynch GS, Hayes A, Campbell SP et al. (1996). Effects of $B_{2}$-agonist administration and exercise on contractile activation of skeletal muscle fibers. Journal of Applied Physiology, 81: 1610-1618.

15. Polla B, Cappelli V, Morello F et al. (2001). Effects of the beta 2agonist clenbuterol on respiratory and limb muscles of weaning rats. American Journal of Physiology, 280: 862-869.

16. Round JM, Matthews $Y$ \& Jones DA (1980). A quick simple and reliable method for ATPase in human muscle preparations. Histochemical Journal, 12: 707-709.

17. Brooke MH \& Kaiser KK (1970). Muscle fiber types: how many and what kind. Archives of Neurology, 23: 369-379.

18. Maltin CA, Hay SM, Delday MI et al. (1987). Clenbuterol, a beta agonist, induces growth in innervated and denervated rat soleus muscle via apparently different mechanisms. Bioscience Reports, 7: 525-532.

19. Stevens L, Gohlsch B, Mounier Y et al. (2000). Upregulation of myosin heavy chain $\mathrm{MHCl} \alpha$ in rat muscles after unweighting and clenbuterol treatment. Biochemical and Biophysical Research Communications, 275: 418-421.

20. Stevens L, Firinga C, Gohlsch B et al. (2000). Effects of unweighting and clenbuterol on myosin light and heavy chains in fast and slow muscles of rat. American Journal of Physiology, 279: C1558-C1563.

21. Kugelberg E (1976). Adaptive transformation of rat soleus motor units during growth. Journal of Neurological Sciences, 27: 269-289. 